\title{
Lipoprotein ratios: Physiological significance and clinical usefulness in cardiovascular prevention
}

\author{
This article was published in the following Dove Press journal: \\ Vascular Health and Risk Management \\ 20 November 2009 \\ Number of times this article has been viewed
}

\begin{abstract}
Jesús Millán'
Xavier Pintó ${ }^{2}$

Anna Muñoz ${ }^{3}$

Manuel Zúñiga ${ }^{4}$

Joan Rubiés-Prat ${ }^{5}$

Luis Felipe Pallardo 6

Luis Masana $^{7}$

Alipio Mangas ${ }^{8}$

Antonio Hernández-Mijares ${ }^{9}$

Pedro González-Santos ${ }^{10}$

Juan F Ascaso"1

Juan Pedro-Botet ${ }^{12}$

'Hospital Universitario Gregorio Marañón, Madrid, Spain; ${ }^{2}$ Hospital Universitario Bellvitge, L'Hospitalet de Llobregat, Barcelona, Spain; ${ }^{3}$ Solvay Pharma, Barcelona, Spain; ${ }^{4}$ Hospital Marqués de Valdecilla, Santander, Spain; ${ }^{5}$ Hospital Vall

d'Hebrón, Barcelona, Spain; ${ }^{6} \mathrm{Hospita}$ Universitario La Paz, Madrid, Spain; ${ }^{7}$ Hospital Sant Joan, Reus, Tarragona, Spain; ${ }^{8}$ Hospital Universitario Puerta del Mar, Cádiz, Spain; ${ }^{\circ}$ Hospital

Universitario Dr Peset, Valencia, Spain ${ }^{10}$ Hospital Clínico Universitario Virgen de la Victoria, Málaga, Spain; "Hospital Clínico Universitario, Valencia, Spain;

${ }^{12}$ Hospital del Mar, Barcelona, Spain
\end{abstract}

Correspondence: Anna Muñoz Solvay Pharma, Av. Diagonal, 507, 08029, Barcelona, Spain

$\mathrm{Tel}+34934954500$

Fax +34934303727

Email anna.munoz@solvay.com

\begin{abstract}
Low-density lipoprotein (LDL) cholesterol concentration has been the prime index of cardiovascular disease risk and the main target for therapy. However, several lipoprotein ratios or "atherogenic indices" have been defined in an attempt to optimize the predictive capacity of the lipid profile. In this review, we summarize their pathophysiological aspects, and highlight the rationale for using these lipoprotein ratios as cardiovascular risk factors in clinical practice, specifying their cut-off risk levels and a target for lipid-lowering therapy. Total/high-density lipoprotein (HDL) cholesterol and LDL/HDL cholesterol ratios are risk indicators with greater predictive value than isolated parameters used independently, particularly LDL. Future recommendations regarding the diagnosis and treatment of dyslipidemia, including instruments for calculating cardiovascular risk or action guidelines, should include the lipoprotein ratios with greater predictive power which, in view of the evidence-based results, are none other than those which include HDL cholesterol.
\end{abstract}

Keywords: apolipoproteins, cholesterol ratios, predictive power, cardiovascular disorders

\section{Introduction}

Estimation of cardiovascular risk has become the cornerstone of cardiovascular prevention. Although atherogenesis is a multifactorial process, abnormalities in lipoprotein metabolism are one of the key factors, representing around $50 \%$ of the population-attributable risk of developing cardiovascular disease. ${ }^{1}$ Despite of the considerable progress made in cardiovascular disease management in recent decades, there is almost unanimous agreement among epidemiologists and clinicians that coronary risk assessment based exclusively on low-density lipoprotein (LDL) cholesterol is not optimal, ${ }^{2}$ particularly in individuals at intermediate risk. ${ }^{3}$ Efforts have been made in seeking emergent or new cardiovascular risk factors to improve cardiovascular disease prediction. ${ }^{1}$ However, it must be emphasized that in an attempt to optimize the predictive capacity of the lipid profile, several lipoprotein ratios or "atherogenic indices" have been defined. These ratios can provide information on risk factors difficult to quantify by routine analyses and could be a better mirror of the metabolic and clinical interactions between lipid fractions. Since lipoprotein ratios are under-used in cardiovascular prevention, but can add to risk assessment, in this review, we briefly summarize their physiological and pathophysiological aspects. Furthermore, we highlight the rationale for using these lipoprotein ratios as cardiovascular risk factors in clinical practice, specifying their cut-off risk levels and a target for lipid-lowering therapy (Table 1). 
Table I Risk categories and target levels for total cholesterol/HDL-C, LDL-C/HDL-C and apoB/apoA-I ratios, in primary and secondary prevention, stratified by gender $6,7,14$

\begin{tabular}{|c|c|c|c|c|c|c|c|c|}
\hline \multirow[t]{3}{*}{ Ratio } & \multicolumn{4}{|c|}{ Primary prevention } & \multicolumn{4}{|c|}{ Secondary prevention* } \\
\hline & \multicolumn{2}{|c|}{ Risk level } & \multicolumn{2}{|c|}{ Target } & \multicolumn{2}{|c|}{ Risk level } & \multicolumn{2}{|c|}{ Target } \\
\hline & Men & Women & Men & Women & Men & Women & Men & Women \\
\hline TC/HDL-C & $>5.0$ & $>4.5$ & $<4.5$ & $<4.0$ & $>4.0$ & $>3.5$ & $<3.5$ & $<3.0$ \\
\hline LDL-C/HDL-C & $>3.5$ & $>3.0$ & $<3.0$ & $<2.5$ & $>3.0$ & $>2.5$ & $<2.5$ & $<2.0$ \\
\hline ApoB/ApoA-I & $>1.0$ & $>0.9$ & $<0.9$ & $<0.8$ & $>0.8$ & $>0.7$ & $<0.7$ & $<0.6$ \\
\hline
\end{tabular}

Note: *Or equivalent risk situation.

Abbreviations: Apo, apolipoprotein; HDL-C, high-density lipoprotein cholesterol; LDL-C, low-density lipoprotein cholesterol; TC, total cholesterol.

\section{Total cholesterol/HDL cholesterol and LDL/HDL cholesterol ratios}

The total/high-density lipoprotein (HDL) cholesterol ratio, known as the atherogenic or Castelli index and the LDL/HDL cholesterol ratio are two important components and indicators of vascular risk, the predictive value of which is greater than the isolated parameters. In this respect, an increase in total cholesterol concentration, and specifically LDL cholesterol, is an atherogenic lipid marker, whereas reduced HDL cholesterol concentration is correlated with numerous risk factors, including the components of the metabolic syndrome, and probably involves independent risk. ${ }^{4}$ When total cholesterol, HDL cholesterol, and total/HDL cholesterol ratio are compared between an apparently healthy population and myocardial infarction survivors, the total/HDL cholesterol ratio is found to present less superposition of populations. ${ }^{5}$ This illustrates the high discriminatory power for coronary heart disease presented by the total/HDL cholesterol ratio, as well as its great predictive capacity. The value of this parameter when the lipid profile is within desirable range should be emphasized. For example, total cholesterol of $231 \mathrm{mg} / \mathrm{dL}(5.89 \mathrm{mmol} / \mathrm{L})$ and HDL cholesterol of $42 \mathrm{mg} / \mathrm{dL}(1.09 \mathrm{mmol} / \mathrm{L})$ gives a total/HDL cholesterol ratio of 5.5, which indicates moderate atherogenic risk. ${ }^{6}$ On the other hand, with the same level of total cholesterol, if HDL cholesterol were $60 \mathrm{mg} / \mathrm{dL}(1.55 \mathrm{mmol} / \mathrm{L})$, the ratio would be 3.8. As total cholesterol/HDL ratio is considered a more sensitive and specific index of cardiovascular risk than total cholesterol, the Canadian working group has chosen this lipid ratio as a secondary goal of theraphy.?

The LDL/HDL cholesterol ratio appears to be as useful as the total/HDL cholesterol ratio. Their similarity can be explained by the fact that approximately two thirds of plasma cholesterol are found in LDL and, consequently, total and LDL cholesterol are closely related. Like the total/HDL cholesterol ratio, LDL/HDL cholesterol may have more predictive power if triglyceridemia is taken into account. ${ }^{8}$ Although, the increase in these ratios predicted a greater cardiovascular risk in a wide range of cholesterol or triglyceride concentrations, the risk is significantly higher when hypertriglyceridemia is present, as shown by the Helsinki Heart Study. ${ }^{9}$

However, it is clear than when there is no reliable calculation of LDL cholesterol, as when triglyceridemia exceeds $300 \mathrm{mg} / \mathrm{dL}$ (3.36 mmol/L), it is preferable to use the total/HDL cholesterol ratio; furthermore, the very-low-density lipoprotein (VLDL) fraction shows cholesterol enrichment in individuals with high triglyceride concentrations, and thus the LDL/HDL cholesterol ratio may underestimate the magnitude of the lipoprotein abnormality in these patients.

Individuals with a high total/HDL cholesterol or LDL/HDL cholesterol ratio have greater cardiovascular risk owing to the imbalance between the cholesterol carried by atherogenic and protective lipoproteins. This may be due to an increase in the atherogenic component contained in the numerator, a decrease in the anti-atherosclerotic trait of the denominator, or both. ${ }^{10}$

Some studies have calculated inverse ratios, ie, HDL/total cholesterol and HDL/LDL cholesterol. Although their predictive value is identical but in the opposite order, few authors suggest they more clearly express the proportion of HDL cholesterol (protective) compared with the rest. ${ }^{8}$

\section{ApoB/ApoA-I ratio}

Apolipoprotein (apo) B represents most of the protein content in LDL and is also present in intermediate-density lipoproteins (IDL) and VLDL. ApoA-I is the principal apolipoprotein in HDL. Both apolipoproteins, therefore, separately provide information for detecting high-risk individuals. ApoA-I is also believed to be a more reliable parameter for measuring HDL than cholesterol content since it is not subject to variation. Therefore, the apoB/apoA-I ratio is also 
highly valuable for detecting atherogenic risk, and there is currently sufficient evidence to demonstrate that it is better for estimating vascular risk than the total/HDL cholesterol ratio. ${ }^{11-14}$ The apoB/apoA-I ratio was stronger than the total cholesterol/HDL holesterol and LDL/HDL cholesterol ratios in predicting risk. ${ }^{11}$ Furthermore, Walldius and colleagues have reported that addition of lipids, lipoproteins or any cholesterol ratio to apoB/apoA-I in risk models did not further improve the strong predictive value of apoB/apoA-I. ${ }^{13}$

This ratio reflects the balance between two completely opposite processes (Figure 1): transport of cholesterol to peripheral tissues, with its subsequent arterial internalization, and reverse transport to the liver. ${ }^{15}$ Figure 2 shows that the greater the apoB/apoA-I ratio, the larger will be the amount of cholesterol from atherogenic lipoproteins circulating through the plasma compartment and likely to induce endothelial dysfunction and trigger the atherogenic process. On the other hand, a lower apoB/apoA-I ratio will lead to less vascular aggression by plasma cholesterol and increased and more effective reverse transport of cholesterol, as well as other beneficial effects, thereby reducing the risk of cardiovascular disease.

\section{Logarithmic transformation of the triglyceride/HDL cholesterol molar concentration ratio}

The association between triglycerides and HDL cholesterol reflected by this ratio depicts the balance between atherogenic and protective lipoproteins. Known as the atherogenic plasma index, it shows a positive correlation with the HDL esterification rate $\left(\mathrm{FER}_{\mathrm{HDL}}\right)$ and an inverse correlation with LDL size. ${ }^{16}$ Therefore, the phenotype of LDL and HDL particles is clearly synchronized with the FER $_{\mathrm{HDL}}$. Hypoalphalipoproteinemia and hypertriglyceridemia both induce an increase in the percentage of small HDL and small, dense LDL particles. Ultimately, the simultaneous use of triglycerides and HDL cholesterol in this ratio reflects the complex interactions of lipoprotein metabolism overall and can be useful for predicting plasma atherogenicity. ${ }^{17}$ An atherogenic plasma index [log (triglycerides/HDL cholesterol)] over 0.5 has been proposed as the cut-off point indicating atherogenic risk. ${ }^{16}$

\section{LDL cholesterol/apoB ratio}

Although apoB is not an apolipoprotein exclusive to LDL, since it is present in other atherogenic lipoprotein fractions such as IDL and VLDL, the LDL cholesterol/apoB concentration ratio provides approximate information on LDL particle size. In this respect, it has been suggested that a ratio of less than 1.3 would indicate the predominance of LDL particles with lower cholesterol content, consistent with small, dense LDL particles. ${ }^{18}$ However, a study comparing LDL particle size determination by gradient-gel electrophoresis with the LDL cholesterol/apoB ratio in healthy and hyperlipidemic subjects failed to find a good correlation and did not recommend the use of this ratio as a surrogate LDL size marker. ${ }^{19}$
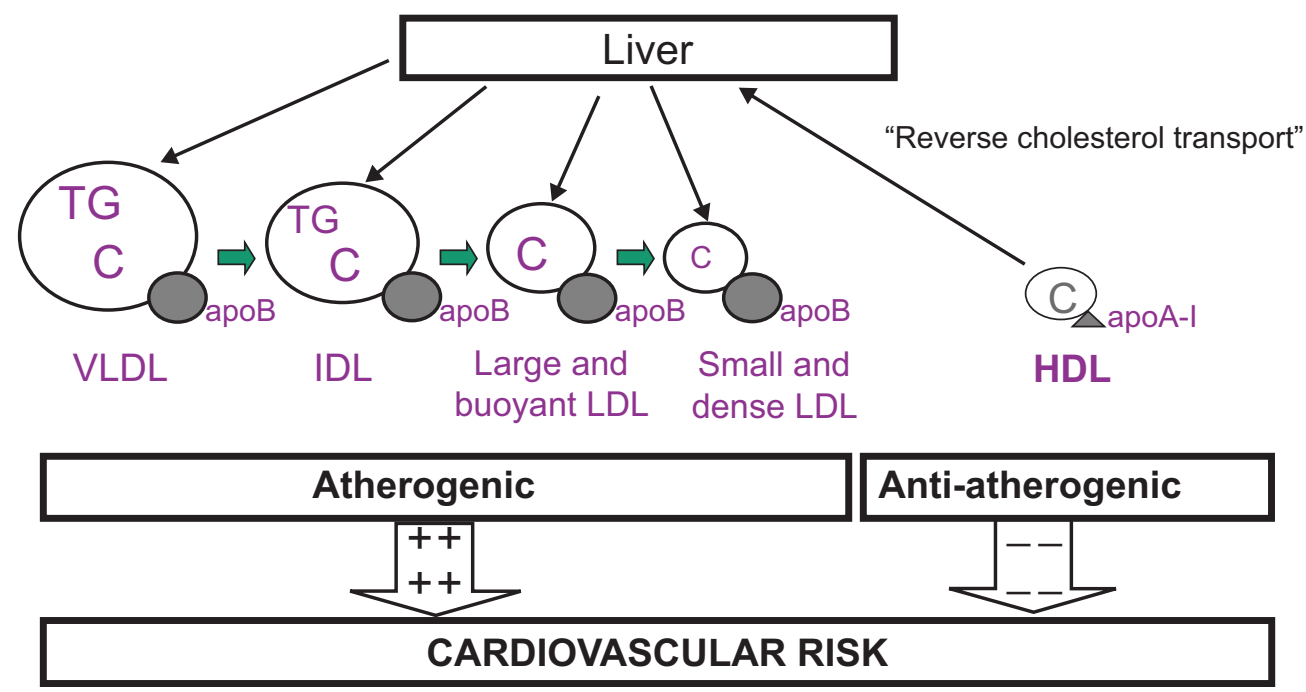

Figure I Atherogenic and anti-atherogenic lipoproteins. This diagram shows that there is one single apolipoprotein B (apoB) molecule in each large, buoyant or small, dense particle of very-low-density (VLDL), intermediate-density (IDL), and low-density lipoproteins (LDL). Therefore, apoB represents the total number of potentially atherogenic particles. Apolipoprotein A-I (apo A-I) is the principal protein component in high-density lipoproteins (HDL) and is responsible for starting reverse cholesterol transport. The balance between apoB and apoA-I is indicative of cardiovascular risk: the greater the ratio, the greater the risk.

Abbreviations: TG, triglycerides; C, cholesterol; ++, increased risk; --, reduced risk. 

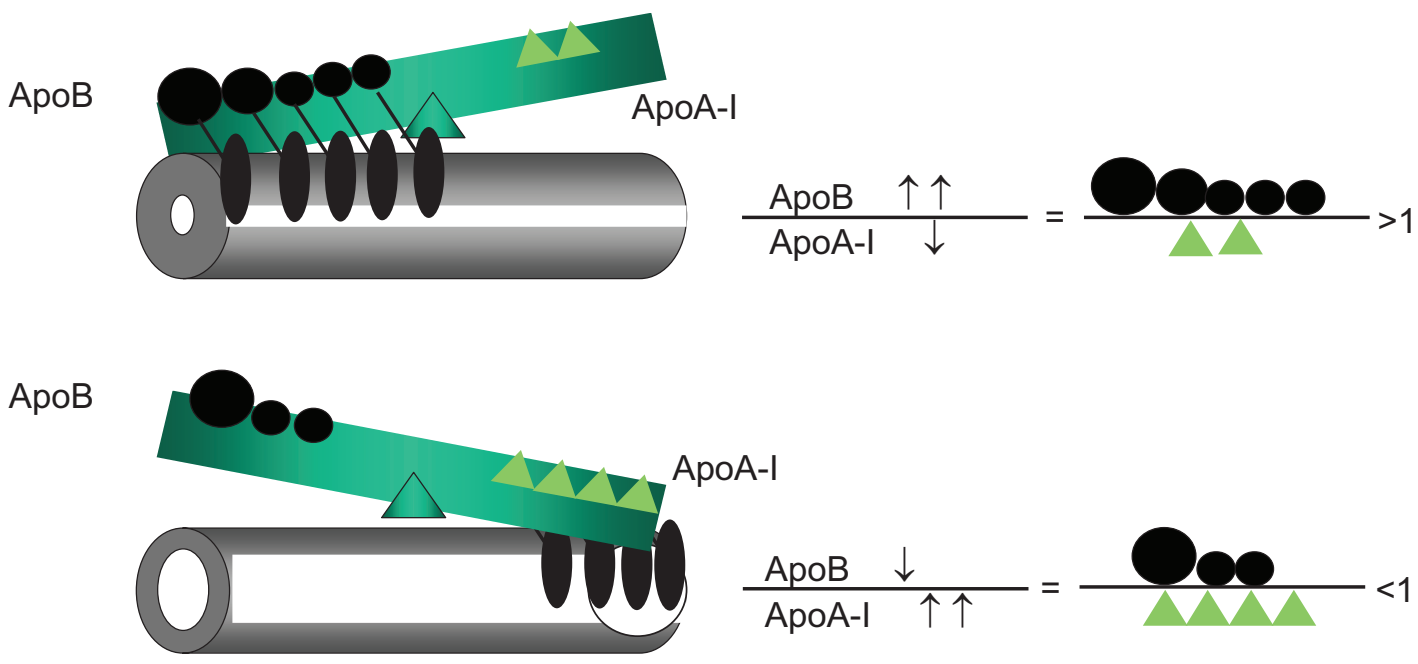

Figure 2 Impact of the apoB/apoA-I ratio on atherogenic balance. Abbreviation: Apo, apolipoproteins.

\section{Non-HDL cholesterol/HDL cholesterol ratio}

Non-HDL cholesterol, which is total cholesterol minus HDL cholesterol, is a measure of the cholesterol in LDL, IDL and VLDL particles. Non-HDL cholesterol has therefore been recommended as a secondary therapeutic target in individuals with high triglyceride concentration, and it has been suggested that it could be a surrogate marker of serum apoB concentration in clinical practice. However, non-HDL cholesterol is not always strongly associated with apoB, particularly in the presence of hypertriglyceridemia. ${ }^{20}$ The non-HDL cholesterol/HDL ratio is a lineal combination of total/HDL cholesterol. Although few studies have evaluated this lipoprotein ratio for predicting cardiovascular disease, and as it is a lineal combination of the results can be assumed to be similar to those of the total/HDL cholesterol or LDL/HDL cholesterol ratios. ${ }^{21}$

\section{Predictive power of lipoprotein ratios for cardiovascular risk}

The evidence derived from large observational studies, including the Framingham Study, ${ }^{22}$ the $\mathrm{LRCP}^{23}$ and the PROCAM, ${ }^{24,25}$ suggests that the total/HDL cholesterol ratio is a more powerful coronary risk predictor than independentlyused total cholesterol, LDL cholesterol and HDL cholesterol. The predictive value for the development of coronary events of the baseline total/HDL cholesterol and LDL/HDL cholesterol ratios in the placebo groups of WOSCOPS, ${ }^{26}$ AFCAPS/ TexCAPS, ${ }^{27}$ and $4 \mathrm{~S}^{28}$ studies was higher than that of other lipid parameters in multivariate analyses. Indeed, the total/HDL cholesterol ratio was included in the Framingham equation to improve risk prediction. ${ }^{29}$ The LRC-CPPT study ${ }^{5}$ analysed the ability of the total/HDL cholesterol ratio to predict coronary risk and compared it to other lipid metabolism variables in three large population-based cohorts: the men in the placebo group of the LRCP-CPPT study; a group of individuals of both sexes subject to follow-up in the Framingham Study; and a group of men and women without coronary disease included in the LRCP Mortality Follow-up Study. The groups of men and women from the Framingham Study and the men from the LRCP-CPPT control group were divided into deciles, using a primary lipid end-point, and the deciles were subsequently divided into terciles using a secondary lipid end-point. When total cholesterol and LDL cholesterol were used as primary end-points, the total/HDL cholesterol and LDL/HDL cholesterol ratios identified significantly high- and low-risk groups within the deciles. For example, when the LRCP-CPPT men were divided into deciles according to LDL cholesterol, the absolute difference in risk within each decile between individuals in the upper and lower terciles of the total/HDL cholesterol ratio was $13 \%$ (95\% confidence intervals [CI]: 8.2\%-17.7\%). The results were similar when the LDL/HDL cholesterol ratio was used. When the LDL/HDL cholesterol and total/HDL cholesterol ratios were used as primary end-points, neither total nor LDL cholesterol identified high- or low-risk groups within the deciles. These results of the stratified analysis were confirmed in the logistic regression analysis and suggest that the total/HDL cholesterol ratio adds risk prediction power to total and LDL cholesterol values. However, no other lipid parameter adds predictive power to the total/HDL 
cholesterol ratio. On the other hand, no conclusive data were obtained in relation to the superiority of the total/HDL cholesterol ratio over the LDL/HDL ratio, or vice versa. Only in women was the total/HDL cholesterol ratio found to have greater predictive power, which could be explained by the fact that this ratio, and not LDL/HDL cholesterol, includes triglyceride-rich lipoproteins in the numerator (VLDL and IDL), and triglycerides are a more powerful coronary risk predictor in women than in men. ${ }^{30}$ When there is no reliable calculation of LDL cholesterol, as occurs in individuals with serum triglycerides above $300 \mathrm{mg} / \mathrm{dL}$ (3.36 mmol/L), since in this scenario the Friedewald formula for estimating LDL cholesterol is not valid, the total/HDL cholesterol ratio will have to be used. The total/HDL cholesterol ratio was the best risk predictor in a subanalysis of the cohort of 14,916 men in the Physicians Health Study ${ }^{31}$ and, after adjusting for other factors, each unit increase in the ratio was found to be associated with a $53 \%$ increase in the risk of myocardial infarction. Similarly, in the cohort of 28,263 postmenopausal women undergoing a three-year follow-up in the Women's Health Study, ${ }^{32}$ among lipid variables, various inflammation markers and homocysteine, the only independent predictors of risk for a cardiovascular event were $\mathrm{C}$-reactive protein and the total cholesterol/HDL cholesterol ratio. Other studies ${ }^{33,34}$ have shown the total cholesterol/HDL cholesterol ratio to be the lipid parameter with the greatest predictive power with regard to the presence, severity and extension of coronary atherosclerosis in women.

The total/HDL cholesterol ratio has also been shown to be a good predictor of carotid intima-media thickness; it has greater power than the isolated variables and similar power to that of the apoB/apoA-I ratio and non-HDL cholesterol. ${ }^{35}$ More controversial is the role of the ratio between the principal protein fractions of LDL and HDL, apoB and A-I, which is the apoB/apoA-I ratio, as contradictory results regarding its use for predicting cardiovascular risk and its relative value compared with the LDL/HDL cholesterol and total/HDL cholesterol ratios have been obtained in the different observational studies. ${ }^{36,37}$ In the Interheart study, which included around 30,000 subjects from 52 countries, the apoB/apoA-I ratio was the variable which predicted a larger proportion of risk of myocardial infarction. Indeed, nearly $50 \%$ of the population-attributable risk corresponded to that ratio. ${ }^{1}$ However, the Interheart study did not include any other lipid measurement, such as HDL or LDL cholesterol, and thus did not compare the relative usefulness of this ratio. In a recent study conducted in 3,322 Framingham inhabitants of both sexes with a 15-year follow-up, the apoB/apoA-I ratio was not superior to the total/HDL cholesterol ratio. ${ }^{38}$ In the EPIC-Norfolk study conducted in apparently healthy men and women, the apoB/apoA-I ratio was associated with the risk of future coronary events independently of the traditional lipid variables, including the total/HDL cholesterol ratio and the Framingham equation. However, the apoB/apoA-I ratio was not a better discriminatory factor between cases and controls than the total/HDL cholesterol ratio, and added little predictive power to the Framingham equation. ${ }^{39}$ Recently, the Cardiovascular Risk in Young Finns Study ${ }^{40}$ has demonstrated that the apoB/apoA-I ratio measured in adolescence was higher than LDL/HDL cholesterol ratio in predicting increased carotid intima-media thickness in adulthood.

Considering these observations and given that apolipoprotein measurement methods are not as widely used as lipoprotein methods and the absence of consensus on reference values and therapeutic objectives, it would appear that the use of apoB/apoA-I ratio is limited for the time being.

Although the total/HDL cholesterol and LDL/HDL cholesterol ratios predict greater cardiovascular risk with a wide range of cholesterol or triglyceride concentrations, the risk is considerably higher in the presence of hypertriglyceridemia. In the Helsinki study, ${ }^{41}$ the LDL/HDL cholesterol ratio was a powerful predictor of cardiovascular risk, mainly in subjects with elevated triglycerides. The greatest risk was observed in subjects with an LDL/HDL ratio $>5$ and triglycerides $>200 \mathrm{mg} / \mathrm{dL}(2.24 \mathrm{mmol} / \mathrm{L})$, who represented around $10 \%$ of the population studied; it was precisely this group which most benefited from treatment with gemfibrozil, with a remarkable $71 \%$ reduction in the incidence of coronary events, more than double that of the overall active-treatment group. In the cohort of 4,559 adult men in the PROCAM observational study, ${ }^{24,25}$ it was found that subjects with an LDL/HDL cholesterol ratio $>5$ presented more than six times the rate of coronary events of those with an LDL/HDL ratio $<5$; however, when the $\mathrm{LDL} / \mathrm{HDL}$ cholesterol ratio was $>5$ and associated with triglycerides $\geq 200 \mathrm{mg} / \mathrm{dL}$ $(2.24 \mathrm{mmol} / \mathrm{L})$, the risk was double that of subjects with triglycerides $<200 \mathrm{mg} / \mathrm{dL}$ ( $2.24 \mathrm{mmol} / \mathrm{L})$. In the Physicians Health Study, ${ }^{31}$ this synergic effect was also found between the increase in the atherogenic/protective cholesterol ratio and hypertriglyceridemia.

\section{Predicting the magnitude of the preventive effect}

The total/HDL cholesterol and LDL/HDL cholesterol ratios are also good predictors of the degree of clinical benefit to be derived from lipid-lowering intervention. ${ }^{10}$ In the LRC-CPPT 
with cholestyramine, the drops in the total/HDL cholesterol ratio in the active treatment group were associated with clinical benefit. In the Helsinki study, multivariate analysis of the relationship between the lipid changes induced by gemfibrozil and the incidence of cardiovascular events in the treated group showed better predictive value for changes in HDL cholesterol and the total/HDL cholesterol ratio. In the placebo group of the AFCAPS/TexCAPS study, ${ }^{27}$ baseline LDL cholesterol had no predictive value for the incidence of coronary events, whereas predictive value was found in HDL cholesterol and the total/HDL cholesterol and LDL/HDL cholesterol ratios, both with greater significance than HDL cholesterol by adjusted logistic regression; in the active treatment group, only changes in apoB and the apoB/apoA-I ratio, which is largely equivalent to the LDL/HDL cholesterol ratio, predicted risk reduction. The total/HDL cholesterol ratio also had more predictive value than other lipid parameters in the control group of the $4 \mathrm{~S}$ study; ${ }^{28}$ treatment-induced changes in this ratio were the best risk predictors in the group receiving simvastatin, with an estimated $17.6 \%$ risk difference for every one-unit reduction in the ratio.

In two angiographic studies in which cholestyramine was used as a hypolipidemiant agent, ${ }^{42,43}$ changes in the total/HDL cholesterol or LDL/HDL cholesterol ratios were the only independent predictors of the benefit observed in coronary stenosis. The predictive capacity of the ratios in this study is also supported by data indicating that an increase in HDL cholesterol is associated with an independent effect on regression, ${ }^{44}$ and that combined treatment with lipid-lowering agents which reduce LDL cholesterol or increase HDL cholesterol more than monotherapy would be associated with a net regression, in comparison with monotherapy, which would only slow down progression. In a meta-analysis of coronary atherosclerosis follow-up studies with intravascular ultrasound scans in patients treated with statins, the apoB/ apoA-I and HDL/LDL cholesterol ratios were powerful predictors of changes in atheroma burden. ${ }^{45}$ This analysis clearly shows that an increase in HDL cholesterol and apoA-I is fundamental, as is a reduction in LDL cholesterol and apoB, for halting progression of coronary atherosclerosis.

At least in secondary prevention, the predictive power of the ratios in relation to the benefit of intervention can be extended to countries with a lower prevalence of coronary disease such as Spain. In a cohort of 882 coronary patients included in the Hospital de Bellvitge Secondary Prevention Programme who were followed up for a mean of 34 months, those who reached the established therapeutic target, an LDL/HDL cholesterol ratio $<3.7$, or reduced ratio by $\geq 15 \%$, reduced their risk of hospitalization for cardiovascular disease and death to less than half, compared with those who did not reach the target. Of the different lipid and nonlipid risk factors, reaching the LDL/HDL ratio objective had the greatest power to predict a favorable outcome in the multiple regression analysis adjusted for possible confounding factors. ${ }^{46}$

\section{What do lipoprotein ratios provide that other conventional risk variables do not?}

Classic cardiovascular risk factors such as age, sex, smoking, diabetes, hypertension, high LDL and low HDL cholesterol levels have been widely analyzed and established as independent cardiovascular risk factors. Many other predictors, such as triglycerides, apoB, lipoprotein(a), homocysteine, C-reactive protein and others have also been studied. Furthermore, the total/HDL cholesterol and LDL/HDL cholesterol ratios are of great interest as major predictors of cardiovascular disease.

Guidelines for lipid management are based on LDL cholesterol concentrations, although other parameters including the total/HDL cholesterol index are better cardiovascular risk predictors than LDL cholesterol and have not been included. ${ }^{47}$ The beneficial use of these indices as important cardiovascular risk predictors is based on multiple epidemiological studies which have shown that these ratios (total/HDL cholesterol and LDL/HDL cholesterol) have a greater correlation with cardiovascular disease and are therefore better predictors of cardiovascular disease than simple lipid parameters. ${ }^{5,31,48}$

A study in 32,826 menopausal women ${ }^{49}$ with an eight-year follow-up, after adjusting for age, smoking, C-reactive protein levels, homocysteine, body mass index, family history, diabetes, hypertension, physical activity, and alcohol consumption, found a relative risk of cardiovascular disease $(95 \% \mathrm{CI})$ of $0.6(0.5-0.8)$ for HDL cholesterol; $1.3(1.0-1.5)$ for triglyceride levels; 1.4 (1.1-1.7) for LDL cholesterol; 1.7 (1.4-2.1) for apoB concentration; 1.6 (1.3-1.9) for nonHDL cholesterol; 1.5 (1.3-1.9) for the LDL/HDL cholesterol index; and 1.6 (1.3-1.9) for the total/HDL cholesterol index. The total/HDL cholesterol index is therefore a good predictor of cardiovascular risk irrespective of other factors.

In a retrospective study with a 20-year follow-up of 1,439 men and 2,812 women with cardiovascular disease, ${ }^{50}$ cardiovascular risk increased in relation to total/HDL cholesterol levels, regardless of total or LDL cholesterol. In men, the relative risk for quintile 5 over 1 of LDL cholesterol 
was 1.85 , whereas the upper over the lower quintile of the total/HDL cholesterol ratio was 2.9. This cardiovascular risk difference between LDL cholesterol and total/HDL cholesterol was not found in women. The authors therefore conclude that men with elevated LDL cholesterol do not have to be treated aggressively if their total/HDL cholesterol index is low, and vice versa, with mild elevations of LDL cholesterol requiring more aggressive treatment if the total/HDL cholesterol ratio is high. This reveals that the total/HDL cholesterol ratio is an excellent cardiovascular risk predictor and a good parameter for deciding on the intensity and need for therapeutic intervention in each different situation.

Other authors ${ }^{51}$ studying subjects with peripheral arterial disease demonstrated the importance of the total/HDL cholesterol index in predicting atherosclerosis in this setting. Studying a cohort of 2,265 subjects with a five-year follow-up, they found disease progression to be significantly related to smoking, the total/HDL cholesterol index and plasma levels of lipoprotein(a) and C-reactive protein.

In an analysis of the Framingham Heart Study and the Coronary Primary Prevention Trial data, ${ }^{52}$ LDL cholesterol and the total/HDL cholesterol and LDL/HDL cholesterol ratios were found to be good cardiovascular disease predictors. However, when vascular changes and cardiovascular risk after intervention were studied, both ratios were better cardiovascular risk reduction predictors than total cholesterol or LDL cholesterol. These ratios should therefore be used when assessing vascular risk and in the reduction of such risk after therapeutic intervention.

There is reason to believe that these lipoprotein ratios, mainly total/HDL cholesterol, based on two values directly measured in a clinical laboratory and which indicate the relationship or proportion between the atherogenic and antiatherogenic lipid fraction, have predictive power for cardiovascular disease and cardiovascular risk reduction after intervention superior to that of classic lipid parameters and should be used in regular clinical practice.

With regard to identifying the "best" lipoprotein ratio, there is an interesting contrast between the use of cut-off points ${ }^{46}$ and quantiles. ${ }^{50}$ Thus it must be taken into account that while cut-off points can provide clinical relevant information, with regard to comparing ratios it would seem more logical to use quantiles within the cohort/population.

\section{Conclusions and recommendations}

LDL cholesterol concentration has been the prime index of cardiovascular disease risk and the main target for therapy. However, data now suggest that total/HDL cholesterol and
LDL/HDL cholesterol ratios are risk indicators with greater predictive value than isolated parameters used independently, particularly the former. These two indices can be regarded as similar; since two thirds of plasma cholesterol are found in LDL, total and LDL cholesterol are closely correlated. The predictive capacity of these ratios is supported by data suggesting that an increase in HDL cholesterol is more prevalently associated with plaque regression, while a decrease in LDL cholesterol would slow down progression. Both predict greater cardiovascular risk for a wide range of cholesterol concentrations. However, when there is no reliable determination of LDL cholesterol, as in cases of hypertriglyceridemia, it is preferable to use the total/HDL cholesterol ratio. This may be particularly interesting in patients with features of the metabolic syndrome.

ApoB constitutes most of the protein content of LDL, as does apoA-I for HDL, even as parameters subject to less variation. Therefore, the apoB/apoAI ratio is also of great value for detecting atherogenic risk and often better than the total/HDL and LDL/HDL cholesterol ratios.

We suggest that future recommendations regarding the diagnosis and treatment of dyslipidemia, including instruments for calculating cardiovascular risk or action guidelines, should include the lipoprotein ratios with greater predictive power which, in view of the results, are none other than those which include HDL cholesterol. Meanwhile, we propose that lipoprotein ratios should be routinely determined to assess the atherogeneity lipids disorders, mainly those characterized by an elevation in plasma triglycerides.

\section{Disclosures}

The authors report no conflicts of interest in this work.

\section{References}

1. Yusuf S, Hawken S, Öunpuu S, et al. Effect of potentially modifiable risk factors associated with myocardial infarction in 52 countries (the INTERHEART study): case-control study. Lancet. 2004;364: 937-952.

2. Superko HR, King S III. Lipid management to reduce cardiovascular risk: a new strategy is required. Circulation. 2008;117:560-568.

3. Arad Y, Goodman KJ, Roth M, Newstein D, Guerci AD. Coronary calcification, coronary disease risk factors, C-reactive protein, and atherosclerotic cardiovascular disease events: the St. Francis Heart Study. J Am Coll Cardiol. 2005;46:158-165.

4. Ascaso J, González Santos P, Hernández Mijares A, et al. Management of dyslipidemia in the metabolic syndrome. Recommendations of the Spanish HDL Forum. Am J Cardiovasc Drugs. 2007;7:39-58.

5. Kinosian B, Glick H, Garland G. Cholesterol and coronary heart disease: predicting risks by levels and ratios. Ann Intern Med. 1994;121: 641-647.

6. Gotto AM, Assmann G, Carmena R, et al. The ILIB lipid handbook for clinical practice: blood lipids and coronary heart disease. 2nd ed. New York, NY: International Lipid Information Bureau; 2000. p. 52, 53, 201. 
7. Genest J, Frohlich J, Fodor G, McPherson R; the Working Group on Hypercholesterolemia and Other Dyslipidemias. Recommendations for the management of dyslipidemia and the prevention of cardiovascular disease: 2003 update. CMAJ. 2003;169:921-924.

8. Pintó X, Ros E. Lípidos séricos y predicción del riesgo cardiovascular: importancia de los cocientes colesterol total/colesterol HDL y colesterol LDL/colesterol HDL. Clin Invest Arterioscl. 2000;12:267-284.

9. Manninen V, Tenkanen L, Koskinen P, et al. Joint effects of serum triglyceride and LDL cholesterol and HDL cholesterol concentrations on coronary heart disease risk in the Helsinki Heart Study. Implications for treatment. Circulation. 1992;85:37-45.

10. Criqui MH, Golom BA. Epidemiologic aspects of lipid abnormalities. Am J Med. 1998;105(Suppl 1A):48S-57S.

11. Walldius G, Junger I, Aastveit A, Holme I, Furberg CD, Sniderman AD. The apoB-apoA-I ratio is better than the cholesterol ratios to estimate the balance between the plasma proatherogenic and antiatherogenic lipoproteins and to predict coronary risk. Clin Chem Lab Med. 2004;42:1355-1363.

12. Sniderman AD, Junger I, Holme I, Aastveit A, Walldius G. Errors that result from using the apoB/apoA-I ratio to identify the lipoprotein-related risk of vascular disease. J Intern Med. 2006;259: 455-461.

13. Walldius G, Junger I. The apoB/apoA-I ratio: a strong, new risk factor for cardiovascular disease and a target for lipid-lowering therapy a review of evidence. J Intern Med. 2006;259:493-519.

14. Holme I, Aastveit AH, Junger I, Walldius G. Relationships between lipoprotein components and risk of myocardial infarction: age, gender and short versus longer follow-up periods in the Apolipoprotein MOrtality RISk study (AMORIS). J Intern Med. 2008;264:30-38.

15. Thompson A, Danesh J. Associations between apolipoprotein B, apolipoprotein $\mathrm{AI}$, the apolipoprotein $\mathrm{B} / \mathrm{AI}$ ratio and coronary heart disease: a literature-based meta-analysis of prospective studies. J Intern Med. 2006;259:481-492.

16. Dobiásová M, Frohlich J. The plasma parameter log (TG/HDL-C) as an atherogenic index: correlation with lipoprotein particle size and esterification rate in apoB-lipoprotein-depleted plasma (FERHDL). Clin Biochem. 2001;34:583-588.

17. Dobiásová M. Atherogenic index of plasma [Log (triglycerides/ HDL-cholesterol)]: theoretical and practical implications. Clin Chem. 2004;50:1113-1115.

18. Vega GL, Beltz WF, Grundy SM. Low density lipoprotein metabolism in hypertriglyceridemic and normolipidemic patients with coronary heart disease. J Lipid Res. 1985;26:115-126.

19. Furuya D, Yagihashi A, Nasu S, et al. LDL particle size by gradient-gel electrophoresis cannot be estimated by LDL-cholesterol/ apolipoprotein B ratios. Clin Chem. 2000;46:1202-1203.

20. Sniderman AD, St-Pierre AC, Cantin B, Dagenais GR, Despres JP, Lamarche B. Concordance/discordance between plasma apolipoprotein B levels and the cholesterol indexes of atherosclerotic risk. Am J Cardiol. 2003;91:1173-1177.

21. Rader DJ, Davidson MH, Caplan RJ, Pears JS. Lipid and apolipoprotein ratios: association with coronary artery disease and effects of rosuvastatin compared with atorvastatin, pravastatin, and simvastatin. Am J Cardiol. 2003;91(Suppl):20C-24C.

22. Castelli WJ, Garrison RJ, Wilson PWF, Abbot RD, Kalousdian S, Kannel WB. Incidence of coronary heart disease and lipoprotein cholesterol levels. The Framingham Study. JAMA. 1986;256:2835-2838.

23. Grover SA, Palmer CS, Coupall L. Serum lipid screening to identify high risk individuals for coronary death. The results of the Lipid Research Clinics prevalence cohort. Arch Intern Med. 1994;154:679-684.

24. Assmann G, Schulte H, Funke H, von Eckardstein A, Seedorf U; The Prospective Cardiovascular Münster (PROCAM) Study. Identification of high-risk individuals for myocardial infarction and the role of HDL. In: Miller NE, editor. High Density Lipoproteins and Atherosclerosis II. Amsterdam, The Netherlands: Elsevier; 1989. p. 51-65.

25. Assmann G, Cullen P, Schulte H. The Munster Heart Study (PROCAM). Results of follow-up at 8 years. Eur Heart J. 1998;19(Suppl A):A2-A11.
26. The West of Scotland Coronary Prevention Study Group. Baseline risk factors and their association with outcome in the West of Scotland Coronary Prevention Study. Am J Cardiol. 1997;79:756-762.

27. Gotto A, Whitney E, Stein EA, et al. Relation between baseline and on-treatment lipid parameters and first acute major coronary event in the Air Force/Texas Coronary Atherosclerosis Prevention Study (AFCAPS/TexCAPS). Circulation. 2000;101:477-484.

28. Pedersen TR, Olsson AG, Faergeman O, et al. Lipoprotein changes and reduction in the incidence of major coronary heart disease events in the Scandinavian Simvastatin Survival Study (4S). Circulation. 1998;97:1453-1460.

29. Anderson KM, Wilson PW, Odell PM, Kannel WB. An updated coronary risk profile. A statement for health professionals. Circulation. 1991;83:356-362.

30. Hokanson JE, Austin MA. Plasma triglyceride level is a risk factor for cardiovascular disease independent of high-density lipoprotein cholesterol level: a meta-analysis of population-based prospective studies. J Cardiovasc Risk. 1996;3:213-219.

31. Stampfer MJ, Krauss RM, Ma J, et al. A prospective study of triglyceride level, low-density lipoprotein particle diameter, and risk of myocardial infarction. JAMA. 1996;276:882-888.

32. Ridker PM, Hennekens CH, Buring JE, Rifai N. C-reactive protein and other markers of inflammation in the prediction of cardiovascular disease in women. N Engl J Med. 2000;342:836-843.

33. Hong MK, Romm PA, Reagan K, Green CE, Rackley CE. Usefulness of the total cholesterol to high-density lipoprotein cholesterol ratio in predicting angiographic coronary artery disease in women. $\mathrm{Am} \mathrm{J}$ Cardiol. 1991;68:1646-1650.

34. Nielsen NE, Olsson AG, Swahn E. Plasma lipoprotein particle concentrations in postmenopausal women with unstable coronary artery disease. Analysis of diagnostic accuracy using receiver operating characteristics. J Intern Med. 2000;247:43-52.

35. Frontini MG, Srinivasan SR, Xu JH, Tang R, Bond MG, Berenson G. Utility of non-high-density lipoprotein cholesterol versus other lipoprotein measures in detecting subclinical atherosclerosis in young adults (The Bogalusa Heart Study). Am J Cardiol. 2007;100:64-68.

36. Sniderman AD, Furberg CD, Keech A, et al. Apolipoproteins versus lipids as indices of coronary risk and as targets for statin treatment. Lancet. 2003;361:777-780.

37. Barter PJ, Ballantyne CM, Carmena R, et al. Apo B versus cholesterol in estimating cardiovascular risk and in guiding therapy: report of the thirty-person/ten-country panel. J Intern Med. 2006;259:247-258.

38. Ingelsson E, Schaefer EJ, Contois JH, et al. Clinical utility of different lipid measures for prediction of coronary heart disease in men and women. JAMA. 2007;298:776-785.

39. van der Steeg WA, Boekholdt SM, Stein EA, et al. Role of the apolipoprotein B-apolipoprotein A-I ratio in cardiovascular risk assessment: a case-control analysis in EPIC-Norfolk. Ann Intern Med. 2007; 146:640-648.

40. Juonala M, Viikari JS, Kähönen M, et al. Childhood levels of serum apolipoproteins B and A-I predict carotid intima-media thickness and brachial endothelial function in adulthood: the Cardiovascular Risk in Young Finns Study. J Am Coll Cardiol. 2008;52:293-299.

41. Manninen V, Tenkanen L, Koskinen P, et al. Joint effects of serum triglycerides and LDL cholesterol and HDL cholesterol concentration on coronary heart disease risk in the Helsinki Heart Study: implications for treatment. Circulation. 1992;85:37-46.

42. Brensike JE, Levy RI, Kelsey SF, et al. Effects of therapy with cholestyramine on progression of coronary arteriosclerosis: results of the NHLBI type II coronary intervention study. Circulation. 1984;69:313-324.

43. Watts GF, Lewis B, Brunt JNH, et al. Effects on coronary artery disease of lipid-lowering diet, or diet plus cholestyramine, in the St. Thomas' Atherosclerosis Regression Study (STARS). Lancet. 1992;339:563-569.

44. Brown BG, Fuster V. Impact of management in stabilization of coronary disease. In: Fuster V, Ross R, Topol EJ, editors. Atherosclerosis and Coronary Artery Disease. Philadelphia, PA: Lippincott-Raven; 1996. p. 191-205. 
45. Nichols Sj, Tuzcu EM, Sipahi I, et al. Statins, high-density lipoprotein cholesterol, and regression of coronary atheroscleorsis. JAMA 2007;297:499-508.

46. Pintó X, Meco JF, Corbella E, et al. Programa de prevención secundaria de la arteriosclerosis de un hospital universitario. Resultados y factores predictivos del curso clínico. Med Clin (Barc). 2003;120:768-772.

47. Hsia SH, Pan D, Berookim P, Lee ML. A population-based, cross-sectional comparison of lipid-related indexes for symptoms of atherosclerotic disease. Am J Cardiol. 2006;98:1047-1052.

48. Ridker PM, Stampfer MJ, Rifai N. Novel risk factors for systemic atherosclerosis: a comparison of C-reactive protein, fibrinogen, homocysteine, lipoprotein(a), and standard cholesterol screening as predictors of peripheral arterial disease. JAMA. 2001;285:2481-2485.
49. Shai I, Rimm EB, Hankinson SE, et al. Multivariate assessment of lipids parameters as predictors of coronary heart disease among postmenopausal women: potential implications for clinical guidelines. Circulation. 2004;110:2824-2830.

50. Nam BH, Kannel WB, D’Agostino RB. Search for an optimal atherogenic lipid risk profile: from the Framingham Study. Am J Cardiol. 2006;97:372-375.

51. Mounier-Vehier C, Stephan D, Aboyans V, et al; Groupe vasculaire (SFC-Societe francaise de medecine vasculaires. The best of vascular medicine in 2006. Arch Mal Coeur Vaiss. 2007;100:47-55.

52. Natarajan S, Glick H, Criqui M, Horowitz D, Lipsitz SR, Kinosian B. Cholesterol measures to identify and treat individuals at risk for coronary heart disease. Am J Prev Med. 2003;25:50-57.
Vascular Health and Risk Management

\section{Publish your work in this journal}

Vascular Health and Risk Management is an international, peerreviewed journal of therapeutics and risk management, focusing on concise rapid reporting of clinical studies on the processes involved in the maintenance of vascular health; the monitoring, prevention and treatment of vascular disease and its sequelae; and the involvement of

\section{Dovepress}

metabolic disorders, particularly diabetes. This journal is indexed on PubMed Central and MedLine. The manuscript management system is completely online and includes a very quick and fair peer-review system, which is all easy to use. Visit http://www.dovepress.com/ testimonials.php to read real quotes from published authors.

Submit your manuscript here: http://www.dovepress.com/vascular-health-and-risk-management-journal 